\title{
Biofeedback fixation training method for improving eccentric vision in patients with loss of foveal function secondary to different maculopathies
}

\author{
Marco U. Morales (1) - Saker Saker • Craig Wilde • Martin Rubinstein • \\ Paolo Limoli • Winfried M. Amoaku
}

Received: 7 May 2019/Accepted: 19 September 2019/Published online: 3 October 2019

(C) The Author(s) 2019

\begin{abstract}
Purpose Fixation stability (FS) of the preferred retinal locus (PRL) may be improved by biofeedback fixation training (BFT) with microperimetry. Such training can be done on the patient's PRL or in different retinal loci with better functional characteristics. We studied both options and compared the outcomes.

Methods Sixty-seven consecutive patients with bilateral central vision loss, poor FS and visual acuity (VA) lower than 0.3 LogMAR were recruited for BFT with microperimeter. Patients were assigned into 2 groups. In group A, BFT was performed on the patient's spontaneous PRL. In group B, PRL was located between 2 adjacent loci with the highest light sensitivity and the lowest distance from the fovea. Two sets of 12 weekly BFT sessions were performed. Primary outcomes were: FS, VA and reading speed.
\end{abstract}

\footnotetext{
M. U. Morales $(\bowtie) \cdot$ S. Saker · C. Wilde .

W. M. Amoaku

Academic Ophthalmology, Division of Clinical

Neurosciences, Queen's Medical Centre, University of

Nottingham, Nottingham NG7 2UH, UK

e-mail: marcoulisesmorales@gmail.com

M. Rubinstein

Department of Optometry, University of Leicester,

Leicester Royal Infirmary, Leicester, UK

P. Limoli

Centro Studi Ipovisione, Milan, Italy
}

Results Outcomes were statistically significantly better in group B. Mean percentage of FS at therapy end improved from 32 to $35 \%$ for group A and from 40 to $55 \%$ in group B. Mean VA improved from 1 to 0.86 in group A and from 1 to 0.84 in group B. Reading speed (wpm) improved from 56 to 58 in group A and from 63 to 89 in group B.

Conclusions This study describes a reliable methodology of improving eccentric fixation stability using BFT in microperimetry, when the fixation training locus is individualized as the retinal area with best functional characteristics. Further studies are needed to validate its value in a larger scale of patients, at different stages of the disease, and its persistence over time.

Keywords Macula - Low vision - Rehabilitation · Retina $\cdot$ Macula $\cdot$ Visual function

\section{Introduction}

Motor neuro-rehabilitation aims to improve patient's functional abilities, replacing skills that have been lost fully or partially. A general neuro-rehabilitation mechanism of action is the potentiation of a group of latent neuronal connections that are utilized repeatedly during challenging behavioural practice. The repeated and persistent practice, over several weeks, of a challenging movement facilitates neural synapsis, 
which may result in lasting physiological changes in motor neural networks [1, 2].

The motor skills acquisition process may be described in distinct phases [2,3], from the early to the consolidation stages, when the newly acquired skill is performed with minimal cognitive resources. The final stage is defined as when performance can be executed after long delays between training sessions [3]. Although the literature on neurological rehabilitation is vast, there are numerous and inconsistent parameters of intensity, frequency and therapy duration related to induced movement studies [1, 4].

The fovea, the centre of the macula, is responsible for detailed vision and fixation. Patients with central vision loss attempt fixation with an eccentric retinal zone known as the preferred retinal locus (PRL) [5]. Macular functional characteristics of light threshold sensitivity, fixation stability (FS) and PRL can be assessed with a microperimetry test, a psychophysical examination similar to static automated perimetry, where patients press a response button when light stimuli are visualized. In addition, a retinal eye-tracker samples and corrects for eye movements, whilst fixation location and FS are plotted as a cloud of fixation points over a reference retinal image [6]. FS in microperimetry is classified as stable, relatively unstable or unstable [7]. The MAIA microperimeter (CenterVue, Italy) scores FS with different indexes; the most representative are $\mathrm{P} 1$ and the bivariate contour ellipse area (BCEA) with proportional values of $95 \%$ [8]. P1 describes the amount of retinal displacement occurring within $1^{\circ}$ from an initial reference point, whilst BCEA describes $95 \%$ of retinal loci used during fixation attempt.

Eyes with eccentric fixation regularly demonstrate unstable FS with associated low vision. However, it has been reported that FS can be improved with oculomotor exercises known as biofeedback fixation training (BFT) [6, 9-13], a task-oriented behavioural therapy, which according to some authors may drive neural plasticity changes in the visual system [11, 14]. BFT consists of asking patients to perform ocular movements towards a specific direction, attempting to align a selected retinal locus with a visual target. This locus is known as the fixation training target. Biofeedback audio signals (beep sounds) aid patients during the oculomotor task by increasing the auditory frequency as the training target approaches the desired alignment.
To be of value, the training target should have better functional characteristics than the PRL previously used by the eye with unstable fixation. However, the selection of this training target has not been previously described.

In this study, we describe a methodology for selecting the best fixation training target, with the aim of improving eccentric fixation through BFT with microperimetry in patients with unstable fixation.

\section{Methods}

This was a prospective, consecutive, case series study of a cohort of patients with irreversible bilateral central vision loss, poor FS and best corrected visual acuity (VA) of 0.3 LogMAR or worse, who performed and completed BFT sessions with the MAIA. The eye with better VA was selected for this study. If both eyes had equal VA, the eye with better FS was chosen. The study included participants from the Low Vision and the Macular Clinics of the Queen's Medical Centre, Nottingham (UK), from January 2013 to June 2017. Local ethical board approval (NRES Committee East Midlands-Nottingham 1) and written informed consent were obtained from all individual participants included in the study, whilst the study met the tenets of the Declaration of Helsinki.

Ninety recruited patients were alternatively divided in 2 different groups. In group A (mean age $64.7 \pm 22$ years), the retinal locus for BFT was set on the baseline patient's spontaneous PRL, assessed with the MAIA Standard-Macula-Test $\left(10^{\circ}, 37\right.$ stimuli). In group B (mean age $70.4 \pm 14$ years), fixation training was set on the locus with the best functional characteristics. To determine this locus, two custom MAIA examinations were performed: (a) the "LowVision-Assessment" grid test $\left(30^{\circ}, 83\right.$ stimuli) with the 4-levels-fixed projection strategy, which scores retinal sensitivity as "good" $(25 \mathrm{~dB})$, "relatively good" (15 dB), "relatively poor" $(5 \mathrm{~dB})$, “poor" $(0 \mathrm{~dB})$ and "scotoma" $(<0 \mathrm{~dB})$; and $(\mathrm{b})$ the "Fixation-Training-Target" grid test $\left(7^{\circ} \times 5^{\circ}, 35\right.$ stimuli) with the 4-2 projection strategy. The first one, (a), was centred on the estimated foveal location or on the patient's PRL in cases of GA larger than 3 times the optic nerve head size (ONH). The Low-VisionAssessment grid output was used to identify retinal loci with at least 2 consecutive stimuli, distributed 
horizontally, showing "good" or "relatively good" light sensitivity, and served as a reference to centre the Fixation-Training-Target grid, prioritizing the superior retina (inferior visual field) and the smaller distance from the anatomical fovea (Fig. 1). The second custom test, (b), is used to select the target locus for BFT. This locus was set in the centre of the two adjacent stimuli with highest light sensitivity and lowest distance from both the anatomical fovea and the baseline PRL.

Adapting the training frequency reported in the literature $[10-12,15,16]$, and with the scope to reach consolidation and retention therapeutic stages, we performed 2 sets of 12 weekly training sessions separated by a 3-month period of no training. Each session lasted $10 \mathrm{~min}$. BFT consisted of asking patients to slightly move their gaze towards the training locus. The auditory signal increased frequency as the desired eye movement reached the target. Patients were asked to remember the gaze movement performed during the training sessions and to try to reproduce the same movement in their daily life when attempting to steadily see a visual target.

Final results were assessed 2 weeks after completing BFT sessions. Primary outcomes were classification of FS, fixation indices P1 and BCEA@95\%, VA, and reading speed (IReST) [17]. FS values after the first training session and after 6 months from baseline were also recorded. Secondary outcomes were mean light sensitivity. The anatomical location and the visual field correspondence of the PRL were also annotated.

Statistical analysis and graphics (GraphPadPrism_7.04) included standard errors, 95\% confidence, interquartile intervals and robust regression outlier removal. Assuming a nonparametric distribution, a one-tailed Mann-Whitney test was applied to compare outcomes between baseline and the last training sessions for each group, and between groups with a significant difference of $p<0.05$. Outcome correlations were analysed through Spearman's rank-order coefficient $\left(r_{\mathrm{s}}\right)$.

\section{Results}

Sixty-seven patients completed the study; 30 had geographic atrophy (GA), 19 moderate dry AMD, 9 Best's disease, 6 myopic macular degeneration, and 3 central serous retinopathy (CSR). Group A included 28 (20 female) and group B 39 (27 female). Mean central scotoma sizes were $5.4^{\circ} \pm 3.8^{\circ}$ and $5.7^{\circ} \pm 4.5^{\circ}$ for groups $\mathrm{A}$ and $\mathrm{B}$, respectively.

At baseline, FS classification in group A was unstable in 18 subjects (64\%), relatively unstable in 9 (32\%) and stable in $1(4 \%)$. At the end of therapy, 16 subjects $(57 \%)$ were classified as unstable, 11 (39\%) as relatively unstable, and 1 (4\%) as stable. In group B, baseline classification was unstable in 19 (49\%) subjects, relatively unstable in 19 (49\%), and

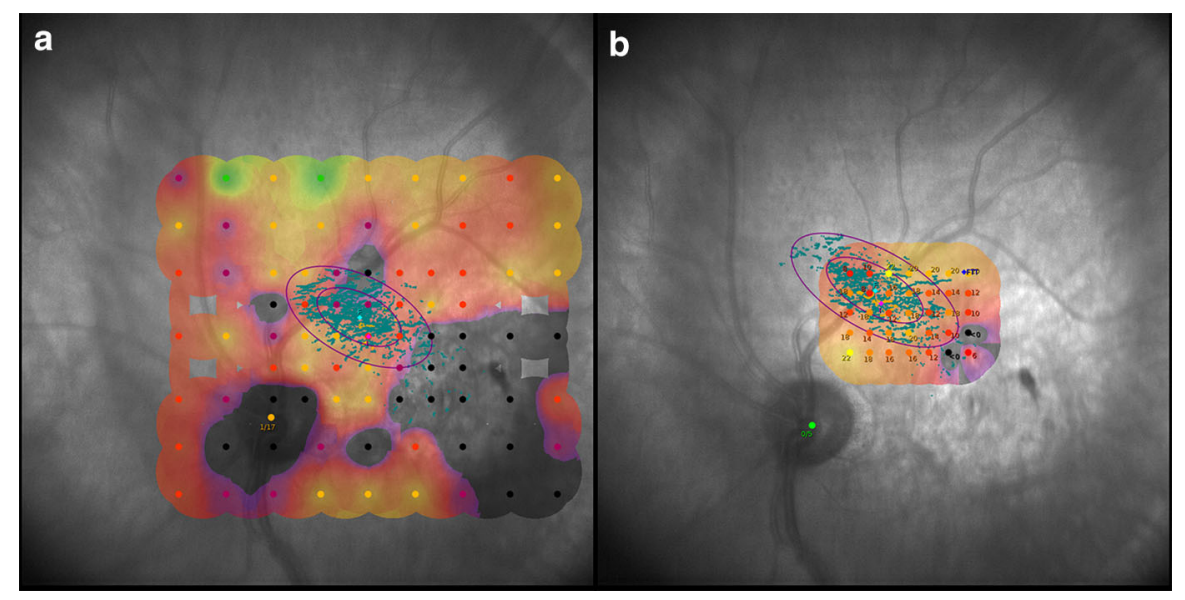

Fig. 1 a "Low-Vision-Assessment" grid centred on patient's PRL with the 4-levels-fixed projection strategy, showing four different $\mathrm{dB}$ levels of intensity, plus absolute scotoma: green $=25 \mathrm{~dB}$, yellow $=15 \mathrm{~dB}$, red $=5 \mathrm{~dB}$, purple $=0 \mathrm{~dB}$, black = scotoma. $\mathbf{b}$ "Fixation-Training-Target" grid with the 4-2 projection strategy, used to select the locus with highest retinal sensitivity for subsequent BFT sessions 
stable in $1(2 \%)$. At therapy end, 11 subjects $(28 \%)$ were classified as unstable, $16(41 \%)$ as relatively unstable, and $12(31 \%)$ as stable (Fig. 2).

In group A, mean FS index P1(\%) was $32 \pm 19$ at baseline, $26 \pm 18$ after the first BFT session, $34 \pm 22$ at 6 months, and $35 \pm 23$ at the end of therapy. In group B, mean P1 was $40 \pm 24$ at baseline, $27 \pm 20$ after the first BFT, $48 \pm 29$ after 6 months, and $55 \pm 29$ at therapy end (Fig. 3).

The mean area $\left(\mathrm{deg}^{2}\right)$ of BCEA@ 95 in group A was $38 \pm 23$ at baseline, $51 \pm 47$ after the first BFT, $33 \pm 22$ at 6 months, and $32 \pm 25$ at study end. In group B, it was $39 \pm 40$ at baseline, $64 \pm 70$ at first BFT, $30 \pm 31$ at 6 months, and $19 \pm 18$ at therapy end (Fig. 3).

FS index P1 did not improve in $50 \%$ of subjects in group A and $18 \%$ in group B. Similarly, $35 \%$ of group A subjects did not improve in BCEA@95\%, compared to $10 \%$ of those from group B.

Mean VA (LogMAR) improvement was observed in $16(57 \%)$ subjects from group A $(1.0 \pm 0.48$ to $0.86 \pm 0.53)$ and $26(67 \%)$ from group B $(1.0 \pm 0.51$ to $0.84 \pm 0.49)$. VA was unchanged in $4(14 \%)$ subjects from group A and 10 (25\%) from group B, whilst a decrease in VA was seen in $8(29 \%)$ participants from group A and $3(8 \%)$ from group B. Mean reading speed (wpm) improved from $56 \pm 30$ to
$58 \pm 32$ in group A and from $63 \pm 36$ to $89 \pm 46$ in group B.

Treatment efficacy (baseline vs therapy end) showed no significant difference in any of the studied variables in group A, as demonstrated with the onetailed Mann-Whitney test shown in Table 1a. In contrast, differences were found in all group B variables except on light threshold sensitivity (LTS) (Table 1b). When comparing final outcomes between groups, a significant difference in all parameters was found, except for VA (Table 1c).

Baseline FS indexes in group A showed moderate correlation $\left(0.50<r_{\mathrm{s}}<0.70\right)$ with their final FS values and scotoma extension. However, high correlation $\left(r_{\mathrm{s}}>0.7\right)$ was found with final reading speed. In group $\mathrm{B}$, baseline FS indexes were highly correlated $\left(r_{\mathrm{s}}>0.7\right)$ with final FS values, whilst moderately correlated with final VA and reading speed. FS outcomes demonstrated a negligible correlation with scotoma size $\left(r_{\mathrm{s}}=0.2\right)$, whilst low dependence with the trained location $\left(r_{\mathrm{s}}=0.3\right)$ was found. Final VA showed better correlation with baseline FS in group B $\left(r_{\mathrm{s}}=0.4\right)$ than in group $\mathrm{A}\left(r_{\mathrm{s}}=0.2\right)$. Final reading speed correlation with FS in group A was high $\left(r_{\mathrm{s}}=0.7\right)$ and moderate $\left(r_{\mathrm{s}}=0.5\right)$ with the trained location, whilst in group B correlation with FS was moderate with FS $\left(r_{\mathrm{s}}=0.5\right)$ and low with the trained location $\left(r_{\mathrm{s}}=0.3\right)$. Low correlation was found
Fig. 2 Classification of fixation stability (FS) on baseline (BL) and end of biofeedback training for both groups $\mathrm{A}$ and $\mathrm{B}$

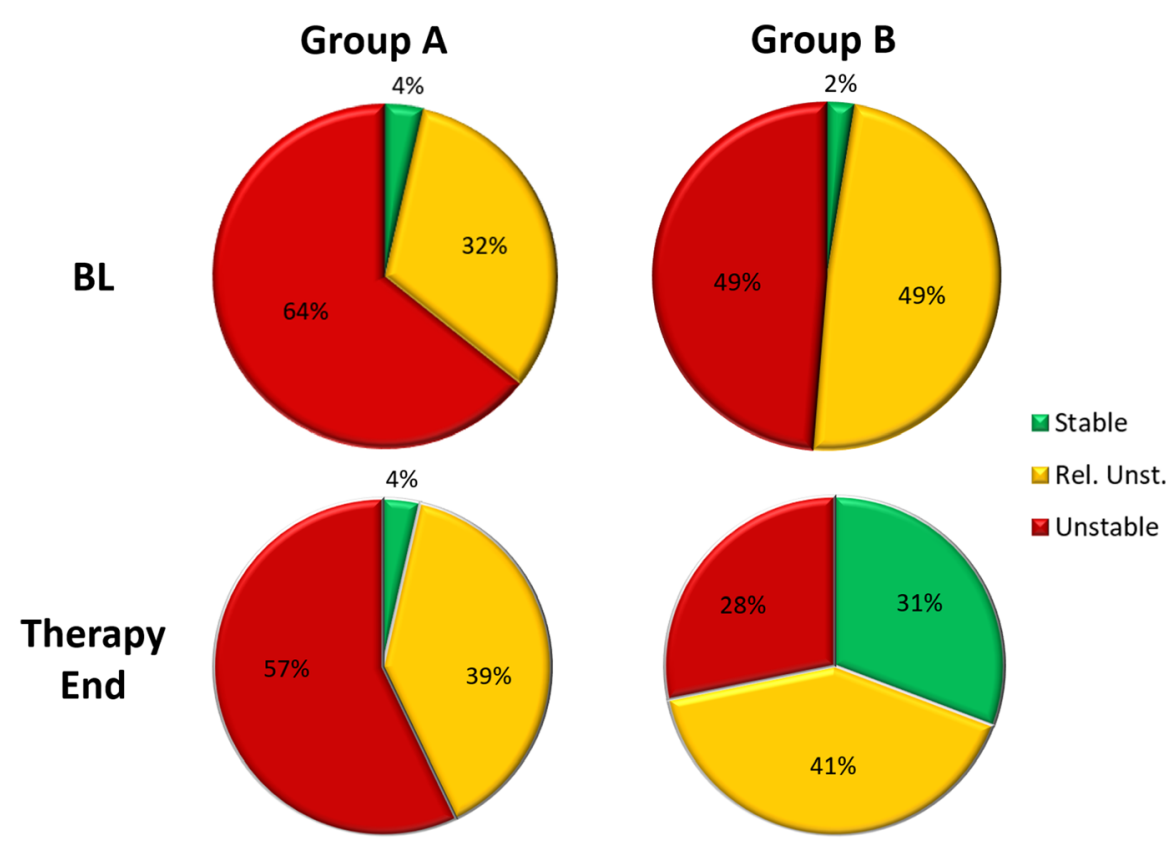


Fig. 3 Box-and-whisker plot showing quartile distribution and mean data of fixation index P1 and BCEA@95\% at baseline (BL), after the first BFT session, 6 months after first treatment and at the end of all BFT sessions (EBFT)
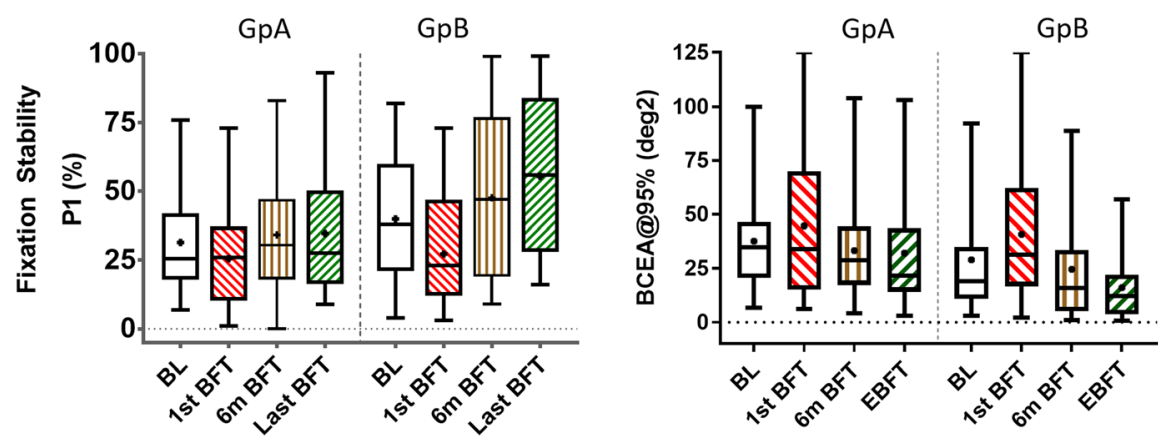

Table 1 Mann-Whitney test of (a) baseline versus therapy end for group A.

(b) Baseline versus therapy end for group B.

(c) Therapy-end comparison for group A versus group B

\begin{tabular}{|c|c|c|c|c|c|}
\hline & $\mathrm{P} 1$ & BCEA@95\% & VA & Reading speed & LTS \\
\hline \multicolumn{6}{|c|}{ (a) GpA $(n=28)$ : baseline versus therapy end } \\
\hline$p$ value & 0.3895 & 0.0734 & 0.1002 & 0.4306 & 0.4115 \\
\hline Significantly different $(p<0.05)$ ? & No & No & No & No & No \\
\hline Mann-Whitney U & 374.5 & 303 & 314 & 381 & 378 \\
\hline Median of baseline & 25.5 & 34.7 & 1 & 49 & 13.25 \\
\hline Median of therapy end & 27.5 & 21.65 & 0.7 & 46.5 & 13 \\
\hline \multicolumn{6}{|c|}{ (b) $G p B(n=39)$ : baseline versus therapy end } \\
\hline$p$ value & 0.0098 & 0.0038 & 0.04 & 0.0078 & 0.1471 \\
\hline Significantly different $(p<0.05)$ ? & Yes & Yes & Yes & Yes & No \\
\hline Mann-Whitney U & 528 & 495 & 586 & 519 & 655 \\
\hline Median of baseline & 38 & 24.7 & 0.92 & 61 & 17.4 \\
\hline $\begin{array}{l}\text { Median of therapy end } \\
\text { (c) GpA versus } G p B \text { therapy end }\end{array}$ & 56 & 13 & 0.7 & 85 & 18 \\
\hline$p$ value & 0.0008 & 0.0035 & 0.4582 & 0.0029 & 0.04 \\
\hline Significantly different $(p<0.05) ?$ & Yes & Yes & No & Yes & Yes \\
\hline Mann-Whitney U & 303 & 336 & 537.5 & 331 & 408.5 \\
\hline Median of GpA, $n=28$ & 27.5 & 21.65 & 0.7 & 46.5 & 13 \\
\hline Median of GpB, $n=39$ & 56 & 13 & 0.7 & 85 & 18 \\
\hline
\end{tabular}

$\left(r_{\mathrm{s}}<0.3\right)$ in both groups between functional outcomes and patient's age. Similarly, no correlation was found $\left(r_{\mathrm{s}}<0.3\right)$ after performing a subgroup analysis to study the PRL behaviour in the different pathologies investigated.

\section{Discussion}

Although task-specific training to enhance motor representations has been reported for several decades [1], only a few authors have demonstrated FS improvement in patients with foveal impairment using biofeedback and microperimetry [9-13, 15, 16, 18, 19]. Furthermore, detailed methodologies adopted to define the best functional retinal locus for such training have not been fully described.

Ramirez et al. [20], following our suggestions, demonstrated the effectiveness of BFT 1 week after completion of therapy, whilst Ratra et al. [21] recently demonstrated in a small number of patients that the BFT effect can be maintained for up to 6 months with a slight reduction in fixation stability. Our study demonstrated a similar reduction, suggesting that such visual training should be attempted for longer periods to achieve maximum results.

Our study adds additional credence to the notion that fixation in patients with eccentric vision can be improved through biofeedback therapy (Fig. 4). Nudo 
Fig. 4 Example of a group B patient, showing the cloud of fixation points with different PRL location and improvement of fixation stability from baseline (a), to end of biofeedback training (b). Fixation improved from unstable to relatively unstable and visual acuity from 1.03 to 0.8 LogMAR

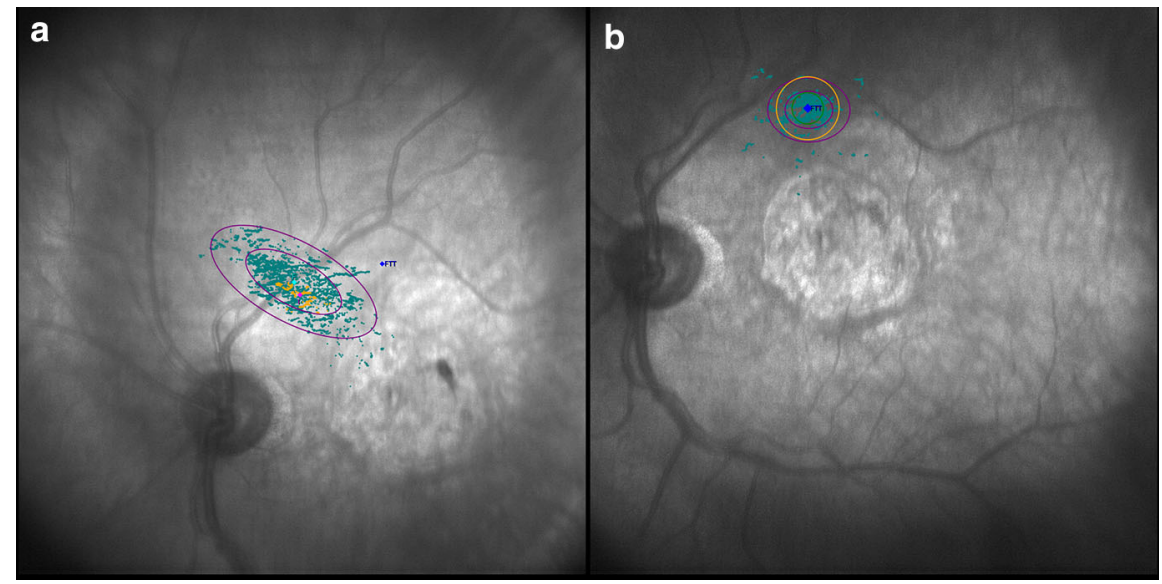

[2] suggested that without behavioural training, plasticity in spared motor areas, which occurs spontaneously, largely reflects the development of compensatory motor patterns rather than patterns of true recovery. Our findings highlight the concept that localized fixation training may enhance plasticity more efficiently than when training is performed on the PRL which was spontaneously developed by the individual after the loss of foveal function.

BFT is reported to be dependent on the location, with the highest retinal sensitivity in small central scotomata [22]. However, we explored the possibility of standardized BFT in cases with any central scotoma size.

The BFT theory is based on neuro-plasticity, where healthy neural sensors are frequently stimulated. When retina photoreceptors and ganglion cells are healthy, microperimetry outcomes demonstrate high light threshold sensitivity values. For this reason, our first FTT selection criteria are a retinal location with good light sensitivity.

Detailed vision is performed with high packing density of cone photoreceptors. Its density peak, located at the foveal centre, decreases rapidly within the central $2 \mathrm{~mm}$, with a gradual decrease further away [23]. Subjects with healthy vision perform fixation within the central $2^{\circ}$, as demonstrated with the MAIA [8]. Consequently, the assumed correlation between density of cones and fixation abilities is valid. Recent studies confirmed photoreceptor's density decreases at $1^{\circ}, 2^{\circ}, 4$ and $6^{\circ}$ of eccentricity, showing a homogenous drop in each of the four retinal meridians, and high agreement between nasal and temporal locations $[23,24]$. These results suggest that patients may have similar anatomical visual capabilities at any retinal meridian with eccentric equidistance from the fovea. In light of these associations, the second FTT selection criterion corresponds to an area located closer to the anatomical fovea without discrimination of the retinal meridian.

Previous studies suggest that reading with eccentric viewing may be more efficient if the PRL is located on the left hemisphere and the lower visual field [25-27]. These observations reinforced our third FTT selection criterion, suggesting the predilection on the left and/or superior side of the central scotoma whenever good light sensitivity is present.

Finally, it is well known that PRL positions may also depend on the visual task. In the western world, reading is performed from left to right. Reading tasks involve eye fixation and saccadic movements following a horizontal path. For that reason, our methodology locates the training location in the middle of a horizontal line, with at least 2 adjacent stimuli with good sensitivity.

Our investigation contributes to the literature with a thorough BFT analysis and scope to understand the rationale behind the selection process of an effective retinal locus useful during eccentric fixation training in patients with foveal function loss. This methodology is summarized as follows:

1. Perform the "Low-Vision-Assessment" grid test with the 4-levels-fixed projection strategy centred on the anatomical foveal or on the patient's baseline PRL in eyes with Geographic Atrophy larger than 3 times the optic nerve head $(\mathrm{ONH})$. 
2. Identify loci with at least 2 consecutive stimuli, distributed horizontally, of good or relatively good threshold sensitivity (GTS).

3. Perform the "Fixation-Training-Target" grid test with the 4-2 projection strategy. Centre the grid on the GTS loci. If there are more than 1 GTS option, prioritize the smaller distance to the fovea on either the superior retina or the left visual field with lower distance from the baseline PRLs.

4. Use the "Fixation-Training-Target" grid outcomes to select the final trained retinal locus to perform biofeedback training (BFT). This locus should be set in the centre of the 2 highest horizontal adjacent threshold stimuli.

5. Perform 10-min BFT sessions over the selected training target on a weekly basis for 12 weeks. After a resting period of 3 months, perform a new set of 12 weekly BFT sessions to aid visual plasticity consolidation.

To conclude, in this study we have described a methodology for biofeedback training with microperimetry, with the scope to improve eccentric vision through better fixation control. Further studies are needed to validate the effectiveness of this methodology in everyday visual tasks, such as reading and other visuomotor activities. Of paramount importance is an investigation of the different motorsequence adaptation stages during BFT, in particular, the recognition of the consolidation and automatic stages, as these may be the key to optimizing frequency and duration for individual therapeutic strategies, as well as to understanding whether longterm plasticity changes can be achieved and retained.

\footnotetext{
Author's contribution MUM designed the study, executed the ophthalmological work, analysed, interpreted the data, wrote the manuscript and is responsible for overall content. SS executed the ophthalmological work, interpreted the data and revised the manuscript. CW assisted in data interpretation and manuscript writing. MR implemented the study and revised the manuscript. PL contributed to design and revised the manuscript. WMA is responsible for overall content, contributed to the study design and wrote and revised the manuscript.
}

Funding This work was carried out as part of the University of Nottingham Ph.D. research program of Marco U. Morales. CenterVue, Padua, Italy, loaned the MAIA microperimeter for the research period.

\section{Compliance with ethical standards}

Conflict of interest Marco Morales was consultant for CenterVue (Padua, Italy). No conflicting relationship exists for the other authors.

Ethical approval All procedures performed in studies involving human participants were in accordance with the ethical standards of the institutional and/or national research committee (NRES Committee East Midlands-Nottingham 1, 12/EM/0116) and with the 1964 Helsinki declaration and its later amendments or comparable ethical standards.

Informed consent This research involved human participants, so an Ethics committee approval and participants' informed consent were obtained.

Open Access This article is distributed under the terms of the Creative Commons Attribution 4.0 International License (http:// creativecommons.org/licenses/by/4.0/), which permits unrestricted use, distribution, and reproduction in any medium, provided you give appropriate credit to the original author(s) and the source, provide a link to the Creative Commons license, and indicate if changes were made.

\section{References}

1. Lang CE, Lohse KR, Birkenmeier RL (2015) Dose and timing in neurorehabilitation: prescribing motor therapy after stroke. Curr Opin Neurol 28(6):549-555. https://doi. org/10.1097/wco.0000000000000256

2. Nudo RJ (2013) Recovery after brain injury: mechanisms and principles. Front Hum Neurosci 7:887. https://doi.org/ 10.3389/fnhum.2013.00887

3. Doyon J, Benali H (2005) Reorganization and plasticity in the adult brain during learning of motor skills. Curr Opin Neurobiol 15(2):161-167. https://doi.org/10.1016/j.conb. 2005.03.004

4. Gee BM, Gerber LD, Butikofer R, Covington N, Lloyd K (2018) Exploring the parameters of intensity, frequency, and duration within the constraint induced movement therapy published research: a content analysis. NeuroRehabilitation 42(2):167-172. https://doi.org/10.3233/nre172233

5. Crossland MD, Engel SA, Legge GE (2011) The preferred retinal locus in macular disease: toward a consensus definition. Retina 31(10):2109-2114. https://doi.org/10.1097/ IAE.0b013e31820d3fba

6. Morales MU, Saker S, Mehta RL, Rubinstein M, Amoaku WM (2013) Preferred retinal locus profile during prolonged fixation attempts. Can J Ophthalmol 48(5):368-374. https:// doi.org/10.1016/j.jcjo.2013.05.022

7. Fujii GY, de Juan E, Sunness J, Humayun MS, Pieramici DJ, Chang TS (2002) Patient selection for macular translocation surgery using the scanning laser ophthalmoscope. Ophthalmology 109(9):1737-1744

8. Morales MU, Saker S, Wilde C, Pellizzari C, Pallikaris A, Notaroberto N, Rubinstein M, Rui C, Limoli P, Smolek MK, 
Amoaku WM (2016) Reference clinical database for fixation stability metrics in normal subjects measured with the MAIA microperimeter. Transl Vis Sci Technol 5(6):6-6. https://doi.org/10.1167/tvst.5.6.6

9. Markowitz SN (2006) Principles of modern low vision rehabilitation. Can J Ophthalmol 41(3):289-312. https:// doi.org/10.1139/i06-027

10. Vingolo EM, Cavarretta S, Domanico D, Parisi F, Malagola R (2007) Microperimetric biofeedback in AMD patients. Appl Psychophysiol Biofeedback 32(3-4):185-189

11. Tarita-Nistor L, Gonzalez EG, Markowitz SN, Steinbach MJ (2009) Plasticity of fixation in patients with central vision loss. Vis Neurosci 26(5-6):487-494. https://doi.org/ 10.1017/s0952523809990265

12. Amore FM, Paliotta S, Silvestri V, Piscopo P, Turco S, Reibaldi A (2013) Biofeedback stimulation in patients with age-related macular degeneration: comparison between 2 different methods. Can J Ophthalmol 48(5):431-437. https://doi.org/10.1016/j.jcjo.2013.07.013

13. Morales MU, Saker S, Amoaku WM (2015) Bilateral eccentric vision training on pseudovitelliform dystrophy with microperimetry biofeedback. BMJ Case Rep. https:// doi.org/10.1136/bcr-2014-207969

14. Chung ST (2011) Improving reading speed for people with central vision loss through perceptual learning. Invest Ophthalmol Vis Sci 52(2):1164-1170. https://doi.org/10. 1167/iovs.10-6034

15. Deruaz A, Goldschmidt M, Whatham AR, Mermoud C, Lorincz EN, Schnider A, Safran AB (2006) A technique to train new oculomotor behavior in patients with central macular scotomas during reading related tasks using scanning laser ophthalmoscopy: immediate functional benefits and gains retention. BMC Ophthalmol 6:35. https://doi.org/ 10.1186/1471-2415-6-35

16. Verboschi F, Domanico D, Nebbioso M, Corradetti G, Scalinci SZ, Vingolo EM (2013) New trends in visual rehabilitation with MP-1 microperimeter biofeedback: optic neural dysfunction. Funct Neurol 28(4):285-291

17. Trauzettel-Klosinski S, Dietz K (2012) Standardized assessment of reading performance: the new international reading speed texts IReST. Invest Ophthalmol Vis Sci 53(9):5452-5461. https://doi.org/10.1167/iovs.11-8284

18. Verdina T, Giacomelli G, Sodi A, Pennino M, Paggini C, Murro V, Virgili G, Menchini U (2013) Biofeedback rehabilitation of eccentric fixation in patients with Stargardt disease. Eur J Ophthalmol 23(5):723-731. https://doi.org/ 10.5301/ejo.5000291
19. Tarita-Nistor L, González EG, Mandelcorn MS, Lillakas L, Steinbach MJ (2009) Fixation stability, fixation location, and visual acuity after successful macular hole surgery. Invest Ophthalmol Vis Sci 50(1):84-89. https://doi.org/10. 1167/iovs.08-2342

20. Ramirez Estudillo JA, Leon Higuera MI, Rojas Juarez S, Ordaz Vera ML, Pablo Santana Y, Celis Suazo B (2017) Visual rehabilitation via microperimetry in patients with geographic atrophy: a pilot study. Int J Retina Vitreous 3:21. https://doi.org/10.1186/s40942-017-0071-1

21. Ratra D, Gopalakrishnan S, Dalan D, Ratra V, Damkondwar D, Laxmi G (2018) Visual rehabilitation using microperimetric acoustic biofeedback training in individuals with central scotoma. Clin Exp Optom. https://doi.org/10.1111/ cxo. 12834

22. Ueda-Consolvo T, Otsuka M, Hayashi Y, Ishida M, Hayashi A (2015) Microperimetric biofeedback training improved visual acuity after successful macular hole surgery. J Ophthalmol. https://doi.org/10.1155/2015/572942

23. Song H, Chui TY, Zhong Z, Elsner AE, Burns SA (2011) Variation of cone photoreceptor packing density with retinal eccentricity and age. Invest Ophthalmol Vis Sci 52(10):7376-7384. https://doi.org/10.1167/iovs.11-7199

24. Lombardo M, Serrao S, Ducoli P, Lombardo G (2013) Eccentricity dependent changes of density, spacing and packing arrangement of parafoveal cones. Ophthalmic Physiol Opt 33(4):516-526. https://doi.org/10.1111/opo. 12053

25. Fletcher DC, Schuchard RA (1997) Preferred retinal loci relationship to macular scotomas in a low-vision population. Ophthalmology 104(4):632-638

26. Nilsson UL, Frennesson C, Nilsson SE (2003) Patients with AMD and a large absolute central scotoma can be trained successfully to use eccentric viewing, as demonstrated in a scanning laser ophthalmoscope. Vis Res 43(16):1777-1787

27. Frennesson C, Nilsson SE (2007) The superior retina performs better than the inferior retina when reading with eccentric viewing: a comparison in normal volunteers. Acta Ophthalmol Scand 85(8):868-870. https://doi.org/10.1111/ j.1600-0420.2007.00984.x

Publisher's Note Springer Nature remains neutral with regard to jurisdictional claims in published maps and institutional affiliations. 